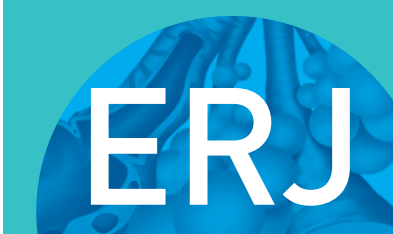

open research
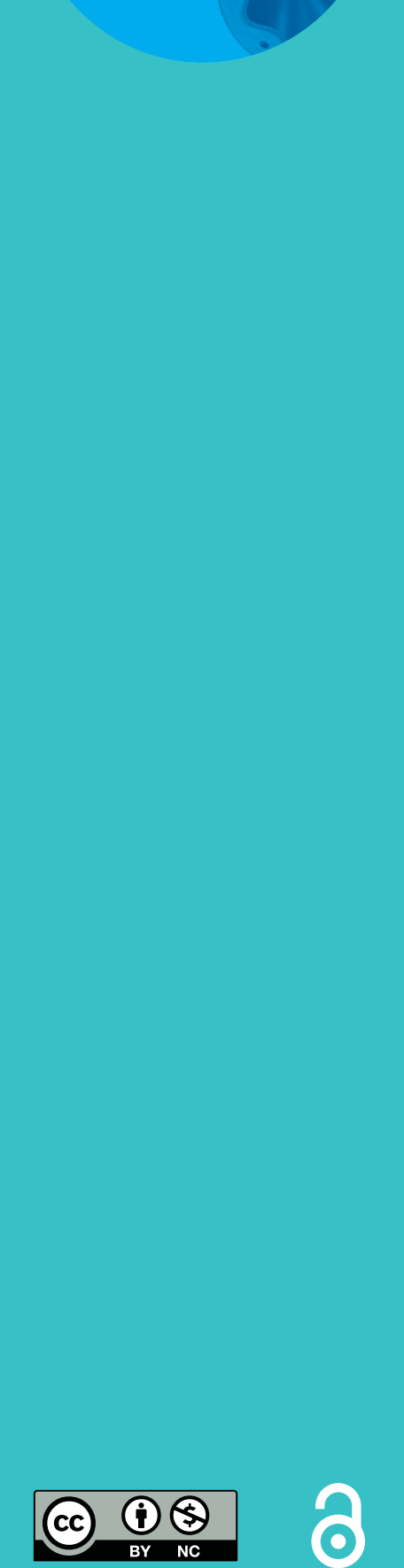

\section{Smoking status and second-hand smoke biomarkers in COPD, asthma and healthy controls}

\author{
Matteo Bradicich ${ }^{1}$ and Macé M. Schuurmans (1) ${ }^{1,2}$
}

Affiliations: ${ }^{1}$ Division of Pulmonology and Sleep Disorders Centre, University Hospital Zurich, Zurich, Switzerland. ${ }^{2}$ University of Zurich, Zurich, Switzerland.

Correspondence: Matteo Bradicich, Division of Pulmonology and Sleep Disorders Centre University Hospital of Zurich, Raemistrasse 100, Zurich, Switzerland. E-mail: Matteo.bradicichQusz.ch

\section{ABSTRACT}

Introduction: Tobacco smoke worsens COPD and asthma. For healthy individuals, quantifying active and second-hand smoke (SHS) exposure clarifies the epidemiology of tobacco consumption and the efficacy of nonsmoking measures. Identifying tobacco exposure biomarkers and cut-offs might allow the creation of sensitive and specific tests.

Aim: We describe the state-of-the-art serum, urinary cotinine and exhaled carbon monoxide (CO) cut-offs for assessing smoking status and SHS exposure in adult patients with COPD or asthma, and healthy controls.

Methodology: After a keyword research in the PubMed database, we included papers reporting on the cut-offs of the investigated biomarkers in one of the populations of interest. Papers published before 2000, not in English, or reporting only data on nonadult subjects or on pregnant women were excluded from the analysis. 14 papers were included in the final analysis. We summarised diagnostic cut-offs for smoking status or SHS exposure in COPD, asthmatic and healthy control cohorts, reporting sensitivity and specificity when available.

Conclusion: Serum and urinary cotinine and exhaled CO are easy-to-standardise, affordable and objective tests for assessing smoking status and SHS exposure. Evidence on cut-offs with good sensitivity and specificity values is available mainly for healthy controls. For COPD and asthmatic patients, most of the currently available evidence focuses on exhaled $\mathrm{CO}$, while studies on the use of cotinine with definite sensitivity and specificity values are still missing. Solid evidence on SHS exposure is available only for healthy controls. An integrated approach with a combination of these markers still needs evaluation.

@ERSpublications

Reliable cut-off values for smoking status in COPD and asthmatic adults are only available for exhaled CO https://bit.ly/34lsHhD

Cite this article as: Bradicich M, Schuurmans MM. Smoking status and second-hand smoke biomarkers in COPD, asthma and healthy controls. ERJ Open Res 2020; 6: 00192-2019 [https:// doi.org/10.1183/23120541.00192-2019].

Received: 6 Aug 2019 | Accepted after revision: 31 March 2020

Copyright $\odot$ ERS 2020. This article is open access and distributed under the terms of the Creative Commons Attribution Non-Commercial Licence 4.0. 


\section{Introduction}

Chronic obstructive pulmonary disease (COPD) and bronchial asthma are obstructive lung diseases that affect together $\sim 900$ million people worldwide [1]. Tobacco smoke plays a predominant role in worsening these conditions. Among the other consequences of primary and secondary tobacco exposure, lung function deterioration and exacerbation rate increase represent the two most epidemiologically and socioeconomically relevant effects in these patients. For healthy individuals and patients, the quantification of tobacco exposure represents an important measure for estimating the epidemiological relevance of tobacco consumption and second-hand smoke (SHS) exposure, and the efficacy of nonsmoking measures and campaigns.

A standardised questionnaire is one of the most popular and widely used tools to assess smoking status. Nevertheless, even though a questionnaire is an easy-to-use, affordable and time-efficient tool, the results might be affected by the recall capacity of the patients. In addition to that, patients might be reluctant to give completely correct answers due to the fear of social judgement associated with smoking. For these reasons, the assessment of smoking status using a questionnaire might be easily affected by subjective judgement and therefore not sufficiently reliable. Furthermore, such assessments might not be completely accurate to determine SHS exposure [2].

Thus, the identification of tobacco exposure biomarkers and the consequent definition of cut-offs represent goals of utmost relevance for establishing adequately sensitive and specific tests.

In this article the state of the art of serum cotinine, urinary cotinine and exhaled carbon monoxide (CO) as biomarkers of smoking status and smoking exposure in adult patients with COPD, asthma and healthy controls will be described.

\section{Search methods}

We searched articles published in PubMed (https://www.ncbi.nlm.nih.gov/pubmed) until March 2019 using the keywords "cotinine", "serum cotinine", "urinary cotinine", "exhaled carbon monoxide", "eCO", "carbon monoxide", "smoking", "smoking status", "smoking cut-off", "smoking exposure", "smoke exposure", "asthma", "chronic obstructive pulmonary disease", "COPD" and "healthy". Papers published before 2000, not in English, or reporting only data on nonadult subjects or only data on pregnant women were excluded from the analysis. 14 papers addressing the topic accordingly to the inclusion criteria were included in our final analysis [2-15]. For each article, we extracted information from the main texts of those reporting data in the abstracts on the cut-offs of at least one of the investigated biomarkers in at least one of the populations of interest.

\section{Results}

\section{Cotinine}

Nicotine and its metabolites are tobacco-specific biomarkers. Nicotine is essentially metabolised in the liver and its half-life is $2-3 \mathrm{~h}$. The half-life of cotinine, one of the main metabolites of nicotine, is $12-20 \mathrm{~h}$. $10-15 \%$ of cotinine is excreted in urine; the rest is metabolised to trans-3'-hydroxycotinine and other byproducts. Because of its pharmacokinetics, cotinine quantification in serum, urine and saliva is commonly used for exposure quantification purposes [2].

\section{Serum cotinine}

Healthy controls

A review by KIM [2] shed light on serum cotinine cut-offs, and showed the best specificity and sensitivity among healthy controls. Considering the shift to the low side in serum cotinine levels among nonsmokers after the establishment of effective tobacco control measures, it is crucial to take into consideration the latest studies about this topic. BENOwitz et al. [4] pointed out how finding the optimal cut-off differentiating smokers from nonsmokers crucially depends on the distribution of cotinine levels between these two populations. Higher cut-offs should therefore be expected in cohorts being exposed to higher levels of SHS, as it was when indoor smoking limitations had not been developed yet.

Among the studies cited in the review by KIM [2], a cut-off of $15 \mathrm{ng} \cdot \mathrm{mL}^{-1}$ presented a sensitivity and a specificity $>95 \%$ in a large population of adult women and men. Nevertheless, the study by BeNowITz et al. [4], conducted on 9901 female and male subjects, showed that a cut-off of $3.08 \mathrm{ng} \cdot \mathrm{mL}^{-1}$ had a sensitivity and a specificity $>95 \%$. As for SHS exposure, BENOwITZ et al. [4] suggested $3 \mathrm{ng} \cdot \mathrm{mL}^{-1}$ as serum cotinine cut-off. Nevertheless, Avila-TANG et al. [3] observed that heavily exposed individuals presented serum cotinine levels up to $25 \mathrm{ng} \cdot \mathrm{mL}^{-1}$; therefore, an overlap might occur. 
COPD patients

SATO et al. [14] reported that serum cotinine concentration correlated positively with the smoking status of COPD patients. Mean \pm SD serum cotinine was $23.2 \pm 69.2$ and $191.1 \pm 109.8 \mathrm{ng} \cdot \mathrm{mL}^{-1}$ in former and current smokers respectively. Moreover, in the COPD population of current smokers, all patients presented serum cotinine concentrations $>50 \mathrm{ng} \cdot \mathrm{mL}^{-1}$. Nevertheless, a statistically significant cut-off value that may distinguish between COPD smokers and nonsmokers has still to be defined.

\section{Asthma patients}

A study by EISNER [7] conducted among 440 nonsmoking adult asthmatic patients and 10581 nonsmoking adult healthy controls showed that there were no statistically significant differences between the median serum cotinine levels of these two groups when exposed to statistically comparable environmental tobacco smoke levels. Both asthmatic and nonasthmatic women in the highest cotinine tertile of the study presented worsened lung function. Even if there were no data about a cut-off that might differentiate between asthmatic smokers and asthmatic nonsmokers, it is plausible that serum cotinine levels were higher in the first group. In the asthmatic population studied by SATO et al. [14], serum cotinine correlated positively with smoking status, although without statistically significant differences. Mean serum cotinine in nonsmokers, former smokers and current smokers was $6.0 \pm 5.2,12.1 \pm 25.0$ and $198.3 \pm 181.7 \mathrm{ng} \cdot \mathrm{mL}^{-1}$, respectively. Data concerning serum cotinine are listed in table 1.

\section{Urinary cotinine}

\section{Healthy controls}

Among the studies cited in the review by KIM [2], two studies by GonIEwICZ et al. [10] and KIM and JUNG [11] showed that a urinary cotinine level between 31.5 and $164 \mathrm{ng} \cdot \mathrm{mL}^{-1}$ correlated with adult healthy controls with a sensitivity $>93 \%$ and a specificity $>95 \%$. The difference in the values might be dependent on the involved population, since the study by KIM and JUNG [11] involved South Korean patients, and that by Goniewicz et al. [10] analysed data from American, Polish and Mexican patients, suggesting that some ethnic and metabolic confounding factors might have played a role [2].

BENOWITZ et al. [4] suggested a urinary cotinine cut-off of $10 \mathrm{ng} \cdot \mathrm{mL}^{-1}$ for SHS exposure among healthy individuals. Furthermore, a study by ZIELINSKA-DANCH et al. [15] estimated more specific urinary cotinine cut-offs for different grades of exposure among smokers and nonsmoker healthy controls. Among smokers, a urinary cotinine level of $2100 \mathrm{ng} \cdot \mathrm{mL}^{-1}$ predicted an elevated daily cigarette consumption. In the nonsmoking population, a urinary cotinine concentration $>170 \mathrm{ng} \cdot \mathrm{mL}^{-1}$ correlated with a high SHS exposure. Furthermore, among nonsmokers with a low SHS exposure, nonsmoking individuals who had essentially no SHS exposure presented a urinary cotinine level $<50 \mathrm{ng} \cdot \mathrm{mL}^{-1}$.

\section{COPD patients}

CHEN et al. [6] detected no statistically significant difference in urinary cotinine levels between COPD patients and healthy controls when both groups were smokers. Both these groups showed higher urinary cotinine levels when compared to healthy controls who were nonsmokers (table 2). A higher urinary cotinine level was associated with greater COPD severity, worsened physical health status and poorer disease-specific quality of life in another study [3].

\begin{tabular}{|c|c|c|c|c|c|c|c|c|}
\hline & \multirow[t]{2}{*}{ First author [ref.] } & \multirow[t]{2}{*}{ Patients } & \multicolumn{3}{|c|}{ Smokers versus nonsmokers } & \multicolumn{3}{|c|}{ SHS } \\
\hline & & & Cut-off $n g \cdot L^{-1}$ & Sns & Spc & Cut-off $n g \cdot L^{-1}$ & Sns & Spc \\
\hline \multirow[t]{2}{*}{$\mathrm{HC}$} & KIM [2] & 824 & 15 & $95 \%$ & $95 \%$ & NA & NA & NA \\
\hline & Benowitz [4] & 9901 & 3.08 & $96.3 \%$ & $97.4 \%$ & 3 & NA & NA \\
\hline COPD & & NA & NA & NA & NA & NA & NA & NA \\
\hline \multirow[t]{2}{*}{ Asthma } & EISNER [7] & 440 & NA & NA & NA & NA & NA & NA \\
\hline & SATo [14] & 170 & NA & NA & NA & NA & NA & NA \\
\hline
\end{tabular}

The study by SATO et al. [14] reported only mean values for each group of patients. Sns: sensitivity; Spc: specificity; SHS: second-hand smoke; NA: not available. 
TABLE 2 Urinary cotinine among nonsmokers, healthy smokers and COPD smokers

\begin{tabular}{lccc} 
& Nonsmokers & Healthy smokers & COPD smokers \\
\hline Subjects & 37 & 40 & 41 \\
Males/females & $19 / 18$ & $35 / 5$ & $38 / 3$ \\
Smoking pack-years & $\mathrm{ND}$ & $21.5 \pm 11.3$ & $30.6 \pm 1.7$ \\
$\boldsymbol{D}_{\text {Lco } \% \text { predicted }}$ & $90.4 \pm 11.4$ & $87.2 \pm 8.5$ & $68.5 \pm 14.3$ \\
Urine cotinine $\mathbf{n g} \cdot \mathbf{m L}^{-1}$ & $\mathrm{ND}$ & $1687.0 \pm 1073.4$ & $1619.5 \pm 885.1$ \\
\hline
\end{tabular}

Data are presented as mean \pm SD unless otherwise stated. $D_{\mathrm{LCO}}$ : diffusing capacity of the lung for carbon monoxide. ND: low or not detected. Reproduced and modified from [6] with permission.

\section{Asthma patients}

FERNANDES et al. [9] assessed a cut-off value for urinary cotinine that was normalised with serum creatinine, in order to take into consideration the influence of renal function on cotinine urinary concentration. The value of $197 \mu \mathrm{g} \cdot \mathrm{g}^{-1}$ confirmed the smoking status previously declared by the patients. Pinheiro et al. [13] showed an increasing trend of creatinine-normalised urinary cotinine values among asthmatic patients with the same smoking status according to the worsening of disease severity. Furthermore, high urinary cotinine concentrations were associated with worsened physical health status and poorer disease-related quality of life [3]. Data concerning urinary cotinine are listed in tables 3 and 4.

\section{Carbon monoxide}

Following smoke inhalation, $\mathrm{CO}$ displaces oxygen in red blood cells, forming carboxyhaemoglobin. In this form, $\mathrm{CO}$ shows a half-life of about 5-6 $\mathrm{h}$ and may be detectable for up to $24 \mathrm{~h}$ depending on ventilation rate, physical activity and sex, among other factors. Tobacco smoke is not the only source of CO, since passive smoking, environmental pollution and occupational exposure might raise CO levels. Nevertheless, tobacco smoke is the most likely cause of high CO levels. In a small number of cases, CO poisoning or acute haemolysis might cause elevated CO levels [12].

\section{Exhaled CO}

Despite showing very good sensitivity and specificity values, cotinine measurements in blood or urine do not allow an immediate assessment of the smoking status since the detection methods require time. In addition, the quantification of serum cotinine requires an invasive procedure. The exhaled CO measurement allows a direct assessment of the specific patient's smoking status using a noninvasive method requiring $<1 \mathrm{~min}$ to perform, with an immediate displayed result.

\section{Healthy controls}

Among healthy controls, the cut-offs for determining the smoking habit of a healthy individual are included in a range that varies from 5 to 6 parts per million $(\mathrm{ppm})[5,12]$. The cut-off value that showed the best sensitivity and specificity combination is $6 \mathrm{ppm}$, with $94 \%$ sensitivity and $96 \%$ specificity. Moreover, exhaled CO showed a positive correlation with the number of smoked cigarettes per day [12]. Exhaled CO was $\sim 1.5$ ppm in healthy nonsmokers; nevertheless, no evidence about a precise cut-off for SHS exposure was provided [8].

TABLE 3 Urinary cotinine concentrations in healthy controls (HC), COPD patients and asthma patients

\begin{tabular}{|c|c|c|c|c|c|c|c|c|}
\hline & \multirow[t]{2}{*}{ First author [ref.] } & \multirow[t]{2}{*}{ Patients } & \multicolumn{3}{|c|}{ Smokers versus nonsmokers } & \multicolumn{3}{|c|}{ SHS } \\
\hline & & & Cut-off & Sns & Spc & Cut-off & Sns & Spc \\
\hline \multirow[t]{2}{*}{$\mathrm{HC}$} & GoNiEWICZ [10] & 601 & $31.5 \mathrm{ng} \cdot \mathrm{mL}^{-1}$ & $97.1 \%$ & $93.9 \%$ & See table 4 & NA & NA \\
\hline & KIM [11] & 11629 & $164 \mathrm{ng} \cdot \mathrm{mL}^{-1}$ & $93.2 \%$ & $95.7 \%$ & NA & NA & NA \\
\hline COPD & Chen [6] & 78 & NA & NA & NA & NA & NA & NA \\
\hline Asthma & FERNANDES [9] & 996 & $196.98 \mu \mathrm{g} \cdot \mathrm{g}^{-1}$ & NA & NA & NA & NA & NA \\
\hline
\end{tabular}

The cut-off showed for asthmatic patients is normalised on serum creatinine, expressed in grams. Sns: sensitivity; Spc: specificity; SHS: second-hand smoke; NA: not available. 


\begin{tabular}{|c|c|c|c|}
\hline & \multicolumn{3}{|c|}{ Cut-off $\mathrm{ng} \cdot \mathrm{mL}^{-1}$} \\
\hline & Patients & Smokers & Exposed nonsmokers \\
\hline Heavy versus light (actual smokers or SHS) & 327 & 2100 & 170 \\
\hline Heavy smokers versus unexposed nonsmokers & 327 & NA & 50 \\
\hline
\end{tabular}

\section{COPD patients}

SATO et al. [14] analysed smoking status in COPD patients who were nonsmokers, former smokers or current smokers, correlating exhaled CO levels with serum cotinine concentrations. The exhaled CO levels in COPD patients were $7.7 \pm 4.3$ and $13.5 \pm 6.5 \mathrm{ppm}$ for former and current smokers respectively (data on nonsmokers were not available). The optimal cut-off to distinguish COPD patients who are current smokers from those who are former smokers or nonsmokers was $11 \mathrm{ppm}$, with $73.1 \%$ sensitivity and $84.7 \%$ specificity. The lower sensitivity and specificity values might be explained by the disease-related inflammatory environment, which may have altered such measurements, as confirmed by EJAZI et al. [8]

As for SHS exposure, an exhaled CO cut-off in COPD patients has still to be determined.

\section{Asthma patients}

SATO et al. [14] compared exhaled CO levels in never-smokers, former smokers and active smokers diagnosed with asthma, correlating these with the serum cotinine concentration. The increase in exhaled $\mathrm{CO}$ reflected the smoking status, reaching the highest level in active smokers and the lowest in never-smokers, with intermediate values for former smokers. The exhaled CO levels in asthmatic patients were $6.1 \pm 2.4,7.7 \pm 3.2$ and $19.9 \pm 17.3 \mathrm{ppm}$, respectively, for never-smokers, former smokers and active smokers. A cut-off for distinguishing current smokers from never-smokers in asthmatic patients was $10 \mathrm{ppm}$, showing $85.0 \%$ sensitivity and $85.8 \%$ specificity. The exhaled CO concentration correlated positively with serum cotinine levels. As for COPD, the lower sensitivity and specificity values might have been affected by the inflammatory environment, which may have altered such measurements.

An exhaled CO cut-off related to SHS exposure in asthmatic patients has still to be defined. Data concerning exhaled $\mathrm{CO}$ are listed in table 5.

\section{Discussion}

Serum cotinine, urinary cotinine and exhaled CO represent efficient methods to define smoking status and to quantify SHS exposure. Among healthy controls, these three biomarkers can predict smoking status with satisfactory sensitivity and specificity values (sensitivity and specificity both $>95 \%$ for serum cotinine, $\geqslant 93 \%$ sensitivity and specificity for urinary cotinine, and $\geqslant 79 \%$ sensitivity and $\geqslant 91 \%$ specificity for exhaled CO). The available data on serum cotinine come from studies that involved a solid number of patients, and reaching comparable results in terms of sensitivity and specificity. The difference between the proposed cut-offs, 15 and $3.08 \mathrm{ng} \cdot \mathrm{mL}^{-1}$, might be due to geographical, ethnic or sex-specific factors, which

\begin{tabular}{|c|c|c|c|c|c|c|c|c|}
\hline & \multirow[t]{2}{*}{ First author [ref.] } & \multirow[t]{2}{*}{ Patients } & \multicolumn{3}{|c|}{ Smokers versus nonsmokers } & \multicolumn{3}{|c|}{ SHS } \\
\hline & & & Cut-off ppm & Sns & Spc & SHS & Sns \% & Spc \% \\
\hline \multirow[t]{2}{*}{$\mathrm{HC}$} & MIDDLETON [12] & 65 & 6 & $94 \%$ & $96 \%$ & NA & NA & NA \\
\hline & Chatrchaiwiwatana [5] & 675 & 5 & $79.4 \%$ & $91.2 \%$ & NA & NA & NA \\
\hline COPD & SATO [14] & 170 & 11 & $73.1 \%$ & $84.7 \%$ & NA & NA & NA \\
\hline Asthma & SATO [14] & 170 & 10 & $85 \%$ & $85.8 \%$ & NA & NA & NA \\
\hline
\end{tabular}


even large patient numbers might have not totally addressed in the cohorts. A similar explanation might be considered for the different urinary cotinine cut-offs. Additionally, urinary cotinine represents an especially flexible and useful tool to assess SHS, since it allows distinction between heavily exposed, lightly exposed and essentially unexposed nonsmokers. The cut-offs for exhaled CO are comparable. The lower sensitivity and specificity values, coming from a 10-fold larger patient cohort [5], are reasonably closer to generalisability. SHS can be predicted as well with these quantification methods, even if some overlap between light smokers and heavily exposed nonsmokers might occur using serum cotinine as a biomarker.

Among COPD patients, the only cut-off currently available to distinguish between smokers and nonsmokers is exhaled CO, with a value of $11 \mathrm{ppm}$ defined from a cohort of 170 patients. Sensitivity and specificity values were remarkably lower compared to those calculated from cohorts of healthy individuals, probably due to chronic lung inflammation processes. Although COPD smokers and nonsmokers can be distinguished by means of exhaled $\mathrm{CO}$, neither exhaled $\mathrm{CO}$ nor the other investigated biomarkers have been strongly associated with SHS exposure. Nevertheless, a higher urinary cotinine level is associated with greater COPD severity, and poorer physical health status and quality of life.

Among asthmatic patients, an exhaled CO cut-off of $10 \mathrm{ppm}$ determined smoking status with good sensitivity and specificity. In a similar way to COPD patients, these values were lower than the ones resulting from the analysis of healthy individual cohorts, probably due to chronic inflammation. Nevertheless, the proposed cut-off showed both higher sensitivity and specificity compared to COPD patients. A possible explanation of this difference might involve years with disease or the age of the subjects, as both these factors affect the inflammatory environment [5] and gas exchange, especially for COPD patients. Even though a specific serum cotinine cut-off to distinguish asthmatic smokers from nonsmokers has not been defined, serum cotinine correlates positively with exhaled CO. There is not much evidence yet for using urinary cotinine to assess smoking status among adult asthmatic patients since well-defined sensitivity and specificity values are still lacking. In this context, the use of normalised urinary cotinine levels by basing it on creatinine excretion has been proposed [11]. A quantification of SHS exposure using these biomarkers is not available among adult asthmatic patients. Nevertheless, high serum and urinary cotinine concentrations correlated with worsened lung function and poorer physical status and quality of life.

\section{Limitations}

The use of these biomarkers has various limitations, mostly concerning metabolising capacities. Different cytochrome P450 (CYP)2A6 expression influences nicotine metabolism and this might affect the smoking status assessment of nonsmoking poor metabolisers and high metabolisers who are current smokers [16]. Moreover, distinct ethnic-specific gene expression might also affect CYP2A6 levels [4]. Specific modifications of the aforementioned cut-offs might be needed also in certain subpopulations of patients receiving therapies that might induce CYP2A6 (e.g. rifampicin, phenobarbital and dexamethasone). Furthermore, in this study, all results regarding pregnant women were excluded due to foreseeable metabolism variations.

Another limitation of this analysis is intrinsically related to cotinine and exhaled CO measurements that, although more objective than questionnaires, could be influenced by clinical, environmental or social settings that may differ among patient populations. For this reason, different cut-offs for different patient populations with the same clinical condition should not be judged a priori as discordant. The timing of measurement in relation to smoke exposure plays an important role but often cannot be influenced. Further studies should assess these issues and address methods for standardisation.

Moreover, some variability of the data relating to SHS exposure may be due to variable definitions of SHS exposure among the cited studies.

Additionally, further research about SHS exposure in adult asthmatic patients is needed, since most of the excluded studies involved asthmatic, nonadult subjects.

\section{Conclusion and future perspectives}

Serum cotinine, urinary cotinine and exhaled CO measurements are readily available tests that may be used in clinical routine with affordable costs. They represent an easy-to-standardise, more objective and reliable method to assess smoking status and SHS exposure than a standardised questionnaire. Evidence on cut-offs with good sensitivity and specificity values is available mainly for healthy controls. For COPD and asthma patients, most of the currently available evidence focuses on exhaled CO, while large studies on the use of cotinine with clearly defined sensitivity and specificity values are still missing. Solid evidence on SHS exposure is available only for healthy controls. Cut-off values based on the current available data have been summarised. 
Future research should be focussed on defining precise cut-offs in order to distinguish different levels of SHS exposure and on assessing such exposure in relation to disease-specific features (e.g. years of disease, exacerbation rate or lung function decline). Moreover, the efficacy of a hierarchical integrated approach (e.g. starting with the most sensible and least invasive test as first-level screening and progressively refining the diagnosis with more specific or expensive tests) has still to be evaluated. The quantification and the pathophysiological effects of third-hand smoke exposure need further in-depth analysis. Additional research for the validation of respective findings among users of electronic cigarettes and heated tobacco products is still needed.

Conflict of interest: M. Bradicich has nothing to disclose. M.M. Schuurmans has nothing to disclose.

\section{References}

1 Vos T, Flaxman AD, Naghavi M, et al. Years lived with disability (YLDs) for 1160 sequelae of 289 diseases and injuries 1990-2010: a systematic analysis for the Global Burden of Disease Study 2010. Lancet 2012; 380: 2163-2196.

2 Kim S. Overview of cotinine cutoff values for smoking status classification. Int J Environ Res Public Health 2016; 13: 1236.

3 Avila-Tang E, Al-Delaimy WK, Ashley DL, et al. Assessing secondhand smoke using biological markers. Tob Control 2013; 22: 164-171.

4 Benowitz NL, Bernert JT, Caraballo RS, et al. Optimal serum cotinine levels for distinguishing cigarette smokers and nonsmokers within different racial/ethnic groups in the United States between 1999 and 2004. Am J Epidemiol 2009; 169: 236-248.

5 Chatrchaiwiwatana S, Ratanasiri A. Exhaled carbon monoxide levels among tobacco smokers by age. Southeast Asian J Trop Med Public Health 2017; 48: 429-437.

6 Chen Q, Deeb RS, Ma Y, et al. Serum metabolite biomarkers discriminate healthy smokers from COPD Smokers. PLoS One 2015; 10: e0143937.

7 Eisner MD. Environmental tobacco smoke exposure and pulmonary function among adults in NHANES III impact on the general population and adults with current asthma. Environ Health Perspect 2002; 110: 765-770.

8 Ejazi MA, Shameem M, Bhargava R, et al. Correlation of exhaled carbon monoxide level with disease severity in chronic obstruction pulmonary disease. Lung India 2018; 35: 401-406.

9 Fernandes AGO, de Souza-Machado C, Pinheiro GP, et al. Dual exposure to smoking and household air pollution is associated with an increased risk of severe asthma in adults in Brazil. Clin Transl Allergy 2018; 8: 48.

10 Goniewicz ML, Eisner MD, Lazcano-Ponce E, et al. Comparison of urine cotinine and the tobacco-specific nitrosamine metabolite 4-(methylnitrosamino)-1-(3-pyridyl)-1-butanol (NNAL) and their ratio to discriminate active from passive smoking. Nicotine Tob Res 2011; 13: 202-208.

11 Kim S, Jung A. Optimum cutoff value of urinary cotinine distinguishing South Korean adult smokers from nonsmokers using data from the KNHANES (2008-2010). Nicotine Tob Res 2013; 15: 1608-1616.

12 Middleton ET, Morice AH. Breath carbon monoxide as an indication of smoking habit. Chest 2000; 117: 758-763.

13 Pinheiro GP, Souza-Machado C, Fernandes AGO, et al. Self-reported smoking status and urinary cotinine levels in patients with asthma. J Bras Pneumol 2018; 44: 477-485.

14 Sato S, Nishimura K, Koyama H, et al. Optimal cutoff level of breath carbon monoxide for assessing smoking status in patients with asthma and COPD. Chest 2003; 124: 1749-1754.

15 Zielinska-Danch W, Wardas W, Sobczak A, et al. Estimation of urinary cotinine cut-off points distinguishing non-smokers, passive and active smokers. Biomarkers 2007; 12: 484-496.

16 Nagano T, Shimizu M, Kiyotani K, et al. Biomonitoring of urinary cotinine concentrations associated with plasma levels of nicotine metabolites after daily cigarette smoking in a male Japanese population. Int J Environ Res Public Health 2010; 7: 2953-2964. 